\title{
Response and encoding factors in "ignoring" irrelevant information
}

\author{
JOHN H. FLOWERS, JACK L. WARNER, and MICHAEL L. POLANSKY \\ University of Nebraska, Lincoln, Nebraska 68588
}

\begin{abstract}
Subjects classified either the numerosity or numeric value of elements in successive stimulus displays. In separate experiments, responses were indicated by oral naming, card sorting, manual tapping, and oral "tapping." Incongruent levels of numeric value slowed naming and sorting, but not tapping, when numerosity was the cue for responding. Incongruent numerosity slowed tapping, but not naming and sorting, when numeric value was the cue. Changes in stimulus response mapping may thus critically alter the ability to ignore an irrelevant stimulus dimension.
\end{abstract}

Historically, the study of human selective attention has emphasized the general selective capabilities of the observer (e.g., Broadbent, 1958; Deutsch \& Deutsch, 1963; Norman, 1968) and to some extent the relation. ship between stimulus structure and the efficiency of selective information extraction (e.g., Garner, 1974). It is becoming increasingly apparent, however, that the susceptibility to interference from irrelevant information can be substantially altered by manipulation of a variety of task and context variables, apart from the stimuli or presumably fixed characteristics of the nervous system. For instance, the presentation of a semantic prime, such as an auditorily presented word, can greatly increase the interference caused by a semantically related word in a color naming task (Posner \& Snyder, 1975; Warren, 1972, 1974). Extensive practice with categorizing a particular semantic class of stimuli can make such targets very difficult to ignore in a subsequent task in which they are irrelevant (Schiffrin $\&$ Schneider, 1977). Findings such as these are increasing the popularity of semantic activation models of attention that place more emphasis upon the tuning and selection of relevant perceptual information (LaBerge, 1976; Posner \& Snyder, 1975) as opposed to the filtering or rejection of irrelevant information emphasized in earlier models (e.g., Broadbent, 1958).

\section{The Role of the Response \\ in Selective Attention}

One task variable that has been underemphasized in selective attention research is that of response structure. Despite the fact that response compatibility has long been recognized as an important factor in human performance, relatively few studies have systematically manipulated response properties, while holding constant

This research was supported by grants-in-aid from the University of Nebraska Research Council, which included funding from NIH Biomedical Research Grant RR-07055-09 to the University of Nebraska. Portions of this paper were presented at the 1976 meeting of the Psychonomic Society. other aspects of the task, to determine the influence of response modality on the interference caused by irrelevant information. This relative neglect may stem from the rather strong distinctions between sensory coding, perception, memory, and response assignment contained in most contemporary information processing models. In such models attentional processes are generally attributed to "earlier" stages than those involved with response selection.

There are, however, some circumstances in which response properties can critically affect interference from irrelevant information. The various multiple stimulation experiments conducted by Greenwald and his associates (e.g., Greenwald, 1970a; Greenwald \& Shulman, 1973) have shown how tasks having relatively compatible relationships between the stimulus and response codes (e.g., orally saying an auditorally presented digit, or manually copying a visually presented digit) were largely immune to disruption by irrelevant but semantically related information. With less compatible relations that required a more complex semantic translation process (e.g., manually copying an auditory digit), considerable interference from competing stimuli was observed.

The literature on the Stroop phenomenon and related tasks also provides examples of how response structure can qualitatively influence selective attention. Such tasks typically exhibit an interference asymmetry; incongruent words disrupt the oral naming of ink colors to a much greater degree than incongruent ink colors interfere with word reading (Dyer, 1973; Stroop, 1935). On the other hand, the directionality of this interference seems to be reversed when the task is one of manual search instead of oral naming. Locating the positions of printed color words is slowed by incongruent ink colors to a greater extent than incongruent words slow the locating of color patches (Uleman \& Reeves, 1971).

Both the Greenwald data and response-dependent interference effects in Stroop-like tasks suggest the existence of semantic information processing domains 
within which the activities of extracting stimulus information and executing responses share common or at least compatible resources. Greenwald (1970a, 1970b) has stressed the importance of ideomotor compatibility-the structural resemblance between the form of stimulus input and the anticipated sensorimotor feedback from response execution. Manually copying a visually displayed digit represents a task with a high degree of ideomotor compatibility, since the spatial structure of the motor response resembles that of the visual display. Such tasks are typically resistant to interference from irrelevant information. An example of a task having a lesser degree of ideomotor compatibility is the copying of an auditory digit. The motor component of the response does not share a structural similarity with the stimulus event. Such tasks are, according to Greenwald, prone to interference from irrelevant information, particularly if the irrelevant information source shares ideomotor compatibility with the response.

While Greenwald's studies stress the importance of the relationship between stimulus and response structure, interpretations of the Stroop phenomenon have focused more upon learned domains of information processing. Treisman and Fearnley (1969) described these semantic domains as perceptual "analyzers," distinguishing between within-analyzer tasks, which keep the information processing requirements within a single domain (reading a word and saying it aloud), and across-analyzer tasks, which require shifting from one domain to another (e.g., reading a word and preparing to match it to a color patch, or sensing a hue and naming it). Within-analyzer tasks are relatively immune to semantic interference effects, whereas across-analyzer tasks tend to be severely disrupted by incongruent information that is already in the domain of the response.

The efficiency of selective attention in semantic conflict tasks is thus dependent upon both structural and learned relationships between stimulus attributes and response activities. The present series of experiments was conducted to determine the extent to which a systematic manipulation of response modalities (holding stimulus properties constant) would qualitatively influence semantic interference from irrelevant information in a speeded classification task. Throughout these experiments, both the stimulus displays and the logical mapping between stimuli (i.e., which stimulus was assigned to which response category) remained invariant; only the mode of executing the response was changed. The specific response modalities chosen were selected to provide a range of both structural similarity and practiced association with the relevant and irrelevant stimulus components.

A somewhat similar study was recently conducted by Beller (1975). In Beller's experiment subjects were required to classify a series of spatial directions (up, down, right, or left) specified either by arrows or by letters ( $U, D, R$, or $\mathrm{L}$ ). Two different response modalities were used: manual tracing and oral naming. When presented with incongruent compounds of these dimensions, incongruent arrow direction severely disrupted tracing the direction specified by the letters, whereas incongruent arrow direction had no significant effect on the oral naming of the letter-specified direction. However, when the subjects had to attend to arrows, the letters interfered regardless of whether the response was oral or manual. Apparently, for that set of stimuli and task, the efficiency of selective attention was not controlled solely by the relative compatibility between each stimulus dimension and response modality; ignoring letters was impossible regardless of the presumed compatibility relationship.

However, there are several aspects of Beller's (1975) study that might have accentuated interference effects to such a degree that any potential asymmetry in interference could have been obscured. For example, as Beller noted, the two different responses required of each subject differed markedly in familiarity and speed of execution; tracing took nearly twice as long as naming. Second, each subject was required to perform under each combination of relevant dimension and response modality, a situation that has been shown in similar tasks to lead to interference effects that do not occur when practice is concentrated on one type of classification task for several trials (Flowers \& Blair, 1976).

The two stimulus dimensions used in the present study (the numeric value of symbols and the numerosity of elements in a display) were chosen largely because of their association with familiar and well practiced perceptual activities (reading and counting). While not equally overlearned, reading and counting tasks produce response times that are more comparable than those for direction naming and tracing. The choice of numeric value and numerosity was further encouraged by the large and reliable Stroop-like interference effects that conflicting values of these dimensions have been shown to produce in naming and classification tasks (Morton, 1969; Windes, 1968). Classification of such incongruent numeric displays could thus be expected to produce unambiguous normative patterns of interference that could be contrasted with data obtained when response properties were systematically manipulated.

\section{EXPERIMENT 1: ORAL NAMING}

The classification of a sequence of displays by orally naming an attribute of each display is the most common version of the Stroop test, and usually the technique that leads to the most compelling interference. The primary purpose of Experiment 1 was thus to provide a normative pattern of data using the same stimulus materials as those to be used in subsequent experiments requiring different response modalities. 


\section{Method}

Subjects. Forty-eight undergraduate volunteers from the introductory psychology course participated in this portion of the study as a means of satisfying a course requirement. Subjects participated individually in a single session lasting 45 to $90 \mathrm{~min}$. All volunteers had normal or corrected acuity and English as their native language.

Stimulus materials. Each stimulus display was typed (SmithCorona Elite) on an $8.9 \times 6.3 \mathrm{~cm}$ white card (longer side vertical) with a small piece cut off the upper left corner to maintain proper orientation. Displays were centered horizontally on these cards and were vertically positioned approximately one-third of the distance from the top edge of the card. During a single experimental trial, subjects responded to a sequence of 36 such cards in a deck.

The stimulus displays contained information about one or both of two stimulus dimensions, numerosity and numeric value. The numerosity dimension was simply the number of discrete elements in a single display, which ranged from one to three. For half the subjects the numeric value dimension was indicated by having the elements appear as digits $(1,2,3)$, while for the remaining subjects numeric value was indicated by words (ONE, TWO, THREE).

Twelve of the subjects who used the digit stimuli were assigned to attend to the numerosity dimension. Two different decks were used for these subjects: a control deck containing one, two, or three asterisks on each card, and an incongruent deck in which one, two, or three repetitions of numerals appeared on each card, arranged such that the numeric value of the digits conflicted with the numerosity of the elements (e.g., 3, 3, 111 , 2, etc.). The asterisks or digits on the cards were spaced approximately $1.0 \mathrm{~cm}$ apart; at the distance most subjects chose to view the stimuli while classifying them, the approximate visual angle subtended by a three-element display was $1.0 \mathrm{deg}$.

Another group of 12 subjects was assigned to attend to numerosity using the word stimuli. Two decks were used for this group, including the asterisk control deck, and an incongruent word deck in which the number words and numerosity were incongruently combined (e.g., ONE ONE ONE, TWO, etc.). Since the words themselves contained between three and five characters, and the spacing between words was $1.0 \mathrm{~cm}$, the visual angle subtended by the arrays had somewhat greater variation and ranged up to about $1.5 \mathrm{deg}$.

The remaining subjects were assigned to attend to numeric value, 12 of them using the digit stimuli and 12 using the word stimuli. Each of these subjects classified three different decks of cards, including a control condition in which numerosity was held constant at one element per card, another control deck for which numerosity was always three elements per card (e.g., 111,222 , or ONE ONE ONE, TWO TWO TWO, etc.), and a deck for which numerosity and numeric value were incongruen tly combined.

Procedure. Before beginning the experiment, each subject was seated at a table and shown examples of the stimulus cards. Those subjects who were assigned to attend to numerosity were instructed that the task required them to orally state the number of elements (words or digits) appearing on each consecutive card, while disregarding the form of the elements. Those subjects who were assigned to attend to numeric value were instructed that the task required them to orally read one of the elements in each consecutive display, while disregarding how many elements appeared on any given card. All subjects were instructed that upon receiving an oral "Teady, set, go" from the experimenter, they were to proceed "as rapidly as possible avoiding errors," dealing off each successive card into a single pile as the display was named. Upon naming the final card, the subjects pressed a key next to the discard pile, which terminated a timing clock. Classification times to the nearest
$.1 \mathrm{sec}$ were recorded and decks were shuffled between trials. The different decks were ordered in blocks of trials, with each block consisting of one trial with each of the decks assigned to that subject. The order of the two decks given to each subject who attended to numerosity was one of simple alternation (with half the subjects beginning with the control deck), while the order of the three decks given to subjects who classified numeric value was counterbalanced among the six possible order permutations. Each subject received two practice blocks, followed by 10 blocks of trials for which data were recorded.

\section{Results and Discussion}

Since uncorrected errors are typically too low in classification tasks of this type to permit meaningful analysis, classification times were used as the sole dependent variable. While these data were recorded as cumulative times for classifying 36 consecutive stimuli, the time values expressed in all tables are converted to mean classification times per stimulus for comparison with studies that used discrete reaction time procedures.

Numerosity relevant (counting). Table 1 presents the mean classification time per stimulus card for the conditions in which numerosity was the cue for responding. For all 12 subjects who classified the digit stimuli, as well as all 12 subjects who classified the word stimuli, naming times for the incongruent stimuli were slower than for the control stimuli. While the size of these interference effects $(50 \mathrm{msec}$ and $37 \mathrm{msec}$ per stimulus for the two types of stimuli) are somewhat less than those reported for color-word interference (see Dyer, 1973), they are both highly reliable and comparable with interference values reported for other types of Stroop naming tasks (e.g., Flowers \& Stoup, 1977). These results are thus consistent with those reported by Windes (1968), demonstrating a substantial disruption of numerosity evaluation by incongruent digits or number words when the response is one of oral naming.

Numeric value relevant (reading). Table 2 displays the mean classification times obtained from those subjects assigned to evaluate numeric value. Since the differences between the classification times for the incongruent stimuli and mean of the two control conditions were only 3 and $6 \mathrm{msec}$ for the digit and word stimuli, respectively, it is apparent that there was no interference effect comparable to that obtained when numerosity was classified. ${ }^{1}$ Informal reports obtained from subjects concur with these data; several

Table 1

Mean Oral Naming Time per Card for Numerosity Dimension in Experiment 1

\begin{tabular}{lcc}
\hline & \multicolumn{2}{c}{ Time } \\
\cline { 2 - 3 } \multicolumn{1}{c}{ Deck } & $\begin{array}{c}\text { Digit } \\
\text { Stimuli }\end{array}$ & $\begin{array}{c}\text { Word } \\
\text { Stimuli }\end{array}$ \\
\hline Control & .493 & .505 \\
Incongruent & .543 & .542 \\
Difference & .050 & .037 \\
\hline
\end{tabular}


Table 2

Mean Oral Naming Time per Card for the Numeric Value Dimension in Experiment 1

\begin{tabular}{lcc}
\hline \multicolumn{1}{c}{ Deck } & \multicolumn{2}{c}{ Time } \\
\cline { 2 - 3 } & $\begin{array}{c}\text { Digit } \\
\text { Stimuli }\end{array}$ & $\begin{array}{c}\text { Word } \\
\text { Stimuli }\end{array}$ \\
\hline One-Item Control & .425 & .433 \\
Three-Item Control & .419 & .427 \\
Incongruent & .425 & .436 \\
Difference* & .003 & .006 \\
\hline
\end{tabular}

*Difference between the incongruent classification time and the mean of the two control conditions.

participants remarked that they were "scarcely aware" of the number of elements on each card.

It is thus apparent that classification by oral naming of these types of numeric displays exhibits an asymmetrical interference effect similar to that found with the naming of colors and color words. Numeric value of words and digits disrupts responding to numerosity, but not the reverse. It should also be noted from a comparison of Tables 1 and 2 that naming of numeric value (reading) produced overall faster times than did the naming of numerosity, which is consistent with a response-compatibility interpretation of the interference effect; the interference occurred in the presence of a distractor that was more compatible with the response than the dimension that was attended. The data from these oral naming tasks thus provide a clear normative pattern against which interference effects obtained with these same stimuli, but using different response modalities, can be evaluated.

\section{EXPERIMENT 2: CARD SORTING}

In speeded classification tasks such as card sorting, there is no obvious structural resemblance between the motor activity required by response and the type of stimulus dimensions used in the present study. Nonetheless, card-sorting tasks have been shown to be subject to interference from irrelevant alphanumeric information (Flowers \& Dutch, 1976; Morton, 1969). Morton (1969) has suggested that although no overt verbalization is required in such tasks, interval covert verbalization may be used as a mediating process in performing the classification; hence the locus of the interference may be similar to that found in overt vocalization tasks. The obtaining of an asymmetric pattern of interference with card sorting identical with that that occurred for the naming tasks would be consistent with the use of such an internal verbal (or numeric) code. However, such a finding would emphasize the need for a somewhat broader interpretation of response compatibility and processing domains than simple structural resemblance between stimulus input and anticipated motor feedback (e.g., Greenwald, 1970b).

\section{Method}

Subjects. Forty-four undergraduates volunteered for a single experimental session lasting 45-60 min. None had participated in Experiment 1. All subjects had normal or corrected acuity and English as their native language.

Stimulus materials. The stimuli consisted of the same decks of cards as used in Experiment 1.

Procedure. Twenty subjects were assigned to sort on the basis of numerosity (10 using the digit stimuli and 10 using the word stimuli). The decks assigned to these subjects were the same as those assigned to the subjects who orally named the numerosity dimension in Experiment 1 . Subjects were told that the task required sorting each of the 36 cards in a deck into three piles according to whether the display on the card contained one, two, or three elements (digits or words). The form of the elements was always to be disregarded.

The remaining subjects were assigned to sort on the basis of numeric value, 12 using the digit stimuli and 12 using the word stimuli. The specific decks assigned were the same as for corresponding conditions in Experiment 1. These subjects were told that the task required sorting the decks into three piles according to the number value of the digit(s) or word(s) on each card; the number of identical digits or words on each card was to be ignored.

Before beginning the experiment each subject was seated at a booth, in which a response key was situated, and was shown examples of the cards to be sorted. Groups of four subjects were run simultaneously in adjacent booths. On each trial a shuffled deck was presented to each subject. The start signal for beginning a trial was provided by projecting on a viewing screen (approximately $3 \mathrm{~m}$ in front of the row of booths) a uniform red field of approximately $.5 \mathrm{sec}$ duration followed immediately by a uniform green field that remained on for $1 \mathrm{sec}$. The red field served as a "ready" signal, while the green signal specified "go." Four digital clocks (one per subject) were activated at the onset of the green field. Subjects were instructed to sort as rapidly as possible avoiding errors, and to press the response key upon sorting the last card in the deck. Deck-sorting times were recorded to the nearest $100 \mathrm{msec}$. Errors were monitored by an experimental assistant who checked each response pile and shuffled the decks for the next trial. Feedback about errors was given to each subject, although as is typical with such tasks, the extremely low rate of uncorrected errors precluded the use of those data in subsequent analysis. Feedback of sorting times was not given.

The order of the two decks given to each subject who sorted according to numerosity was simple alternation, while the order of the three decks given to each subject who sorted according to numeric value was counterbalanced such that all six permutations of the decks within a block occurred twice among the 12 subjects. As in the previous experiment, each subject received 2 practice and 10 experimental blocks of trials.

\section{Results and Discussion}

Table 3 presents the mean sorting times per stimulus averaged across blocks of trials and subjects for classification of numerosity; Table 4 presents similar data for classification of numeric value. Qualitatively, the pattern of data closely resembles that obtained with oral naming. When sorting according to numerosity, subjects produced longer sorting times with the incongruent decks than with the control decks $[F(1,9)=9.91$, MSe $=2.27, p<.025$, for the digit stimuli $F(1,9)=18.3$, $\mathrm{MSe}=1.43, \mathrm{p}<.01$, for the word stimuli $].{ }^{2}$ While the sorting times themselves are comparable with the naming times obtained in Experiment 1, the absolute size 
Table 3

Mean Oral Naming Time per Card for Numerosity Dimension in Experiment 2

\begin{tabular}{lcc}
\hline & \multicolumn{2}{c}{ Time } \\
\cline { 2 - 3 } \multicolumn{1}{c}{ Deck } & $\begin{array}{c}\text { Digit } \\
\text { Stimuli }\end{array}$ & $\begin{array}{c}\text { Word } \\
\text { Stimuli }\end{array}$ \\
\hline Control & .542 & .530 \\
Incongruent & .561 & .550 \\
Difference & .19 & .20 \\
\hline
\end{tabular}

Table 4

Mean Sorting Time per Card for Numeric Value Dimension in Experiment 2

\begin{tabular}{lcc}
\hline & \multicolumn{2}{c}{ Time } \\
\cline { 2 - 3 } \multicolumn{1}{c}{ Deck } & $\begin{array}{c}\text { Digit } \\
\text { Stimuli }\end{array}$ & $\begin{array}{c}\text { Word } \\
\text { Stimuli }\end{array}$ \\
\hline One-Item Control & .492 & .499 \\
Three-Item Control & .479 & .477 \\
Incongruent & .487 & .495 \\
Difference & .002 & .007 \\
\hline
\end{tabular}

of the interference effects are less for sorting than for naming (19 and $20 \mathrm{msec}$ per stimulus as opposed to 50 and $37 \mathrm{msec}$ ). Slightly smaller interference levels for manual classification as opposed to overt naming have also been noted for color-word interference (e.g., Keele, 1972).

As in Experiment 1, there is little or no evidence for interference with numeric value classification by incongruent levels of numerosity, with the sorting times for the incongruent stimuli falling between the times produced by the control decks. Again, verbal reports from subjects who sorted according to numeric value indicated none of the compelling fellings of frustration that typically accompany semantic interference effects; rather vehement reports of such frustration did, however, come from subjects who sorted according to numerosity.

An additional behavioral observation that may have a bearing upon the form of internal coding and source of interference is that subjects, without exception, placed the "ones" pile on the left and "threes" pile on the right, even though no specific instructions were given about pile placement. It seems quite plausible that the use of a numeric positional code, which is semantically closer to the numeric value dimension (in that it can be more efficiently generated from reading than counting) serves as a means of response organization.

Additional evidence for the use of a linearly ordered semantic code of this type comes from a study by Hock and Petrasek (1973), in which a generalization gradient of interference from irrelevant numbers was produced in a task requiring the classification of elipses varying in size. Interference was dependent upon the numeric "distance" between the interfering digit and the ordinal position of elipse size. Thus the term "response compatibility," both in the present case and in Hock and Petrasek's study, should be related more to the generation of a mediating organizational code than to the simple motor act of tossing a card into a pile. Linear ordering would cleraly seem to be an important property of this code.

\section{EXPERIMENT 3: MANUAL TAPPING}

The response modality used in the third experiment was also manual, but was selected to provide a very strong structural resemblance to the numerosity dimension. It involved simple tapping with the point of a felt pen, the number of times indicated by the value of the relevant stimulus dimension, and thus actually constituted a direct representation of numerosity. Presumably, therefore, encoding the numeric value dimension into a form suitable for executing a tapping response would be less efficient than code generation from numerosity itself, a reverse of the compatibility relationships found in the previous two experiments.

\section{Method}

Subjects. Forty-four undergraduate volunteers from the introductory psychology course each served in a single session lasting 45-90 min. None of the subjects had participated in either of the previous experiments. All had normal or corrected acuity and English as the native language.

Stimulus materials. In place of the stimulus cards used in the previous two experiments, stimulus displays were typed in two columns of 18 stimuli each on $27.8 \times 21.5 \mathrm{~cm}$ white paper sheets. However, the stimuli contained in these lists were identical to those printed on the previously used cards, with one list type corresponding to each of the different decks used in the previous experiments (asterisk control, incongruent digit, incongruent word, one-word control, one-digit control, three-word control, and three-digit control). Since the stimuli in a sequence of 36 could not be shuffled, five different lists of each type were constructed. The order of stimuli in the list was randomized with the restriction that no identical display could occur sequentially.

Since the response required subjects to mark next to each display with a felt pen, each list was overlayed with a removable clear acetate sheet prior to the initiation of a trial. This allowed monitoring of errors and reuse of the stimulus sheets.

Procedure. Subjects were run in groups of four using the same apparatus as in Experiment 2. Twenty subjects were assigned to tap on the basis of numerosity $(10 \mathrm{using}$ the digit stimuli, and 10 using the word stimuli). They were instructed to scan vertically down each of the columns, beginning with the leftmost column, making one, two, or three marks with the felt-tip pen immediately to the right of each display, according to the number of elements present. The form of the elements was to be disregarded. Twenty-four subjects were assigned to tap on the basis of numeric value (12 using the digit stimuli and 12 using the word stimuli). These subjects were instructed to scan vertically down each column, tapping one, two, or three marks to the right of each successive display according to the numeric value of one of the digits or words contained in the display. The number of repeated digits or words was to be disregarded. Subjects were instructed to proceed as rapidly as possible avoiding errors, following a start signal identical to that given in Experiment 2, and to press the key following the marking of the last display on the list.

Each subject received 2 practice blocks of trials followed 
by 10 experimental blocks (ordered as in Experiment 2) for which data were recorded.

\section{Results and Discussion}

Table 5 displays the classification times per stimulus for subjects who classified numerosity. No interference effect was observed for either word or digit stimuli; mean "tapping time" per stimulus was actually slightly faster for the incongruent lists. Thus, in contrast to the previous two experiments, subjects in this tapping experiment were able to effectively ignore incongruent levels of numeric value, even though stimulus displays and the logical mapping between stimuli and response categories was essentially identical to those used in the naming and sorting experiments.

For those subjects who classified the numeric value dimension, the outcome was somewhat ambiguous. From Table 6 it can be seen that for the word stimuli, the incongruent lists produced classification times that were about $15 \mathrm{msec}$ longer than the control conditions, and analysis of variance revealed a marginally significant effect of list type $[F(2,22)=5.11$, MSe $=1.98$, $\mathrm{p}<.05]$. Furthermore, the incongruent condition produced the slowest classification time for 11 of 12 subjects. However, evidence for an interference effect with the digit stimuli is less compelling; no significant effect of list type was noted $[\mathrm{F}(2,22)=.86$, $\mathrm{MSe}=2.51]$. It should be noted, however, that the tapping task produced a considerably greater amount of variability in classification times between trials than occurred for sorting, as evidenced by the fact that the MSe times are considerably larger. This likely reflects the unpracticed nature of tapping with a felt pen as opposed to sorting cards. We thus decided to replicate a numeric value classification task using a tapping

Table 5

Mean Classification (Tapping) Time per Stimulus for Numerosity Dimension in Experiment 3

\begin{tabular}{lcc}
\hline & \multicolumn{2}{c}{ Time } \\
\cline { 2 - 3 } List Type & $\begin{array}{c}\text { Digit } \\
\text { Stimuli }\end{array}$ & $\begin{array}{c}\text { Word } \\
\text { Stimuli }\end{array}$ \\
\hline Control & .499 & .547 \\
Incongruent & .498 & .541 \\
Difference & -.001 & -.006 \\
\hline
\end{tabular}

Table 6

Mean Classification (Tapping) Time per Stimulus for Numeric Value Dimension in Experiment 3

\begin{tabular}{lcc}
\hline & \multicolumn{2}{c}{ Time } \\
\cline { 2 - 3 } \multicolumn{1}{c}{ List Type } & $\begin{array}{c}\text { Digit } \\
\text { Stimuli }\end{array}$ & $\begin{array}{c}\text { Word } \\
\text { Stimuli }\end{array}$ \\
\hline One-Item Control & .548 & .561 \\
Three-Item Control & .549 & .562 \\
Incongruent & .554 & .576 \\
Difference & .005 & .015 \\
\hline
\end{tabular}

response under conditions that we felt might lead to more stable data.

\section{EXPERIMENT 4: MANUAL TAPPING CONTINUED}

Even if the apparently slower numeric value classification times observed for the incongruent stimuli in Experiment 3 constitute a "real" effect, there remains an additional problem in interpretation. Actual observation of subjects performing the tapping indicated a tendency to follow the contour of the displays in each column for those conditions in which numerosity of elements varied. For those control conditions that held numerosity constant at one or three elements, no such contour existed; that is, the columns were straight. Since it was conceivable that, for this mode of response execution, the positional uncertainty of the incongruent displays might have slowed classification times apart from any interference effect, it was decided to use a more conservative control condition (one that incorporated an equivalent amount of positional uncertainty) in the replication. Other modifications of the procedure were designed to provide conditions that were more conducive to stable performance across trials and subjects. These included the provision of more practice by collection of data over two separate sessions, the reduction of the number of different list types given to each subject from three to two, and the running of subjects individually instead of in groups of four.

\section{Method}

Subjects. Twenty undergraduates from the introductory psychology course each served in two sessions on successive days. Each session lasted approximately $30 \mathrm{~min}$. All subjects had normal or corrected acuity, English as their native language, and had not participated in any of the previous experiments.

Procedure. All 20 subjects classified the stimulus displays according to numeric value, 10 using digit stimuli and 10 using word stimuli. As in the previous study, the stimulus displays were arranged in two columns on a white paper sheet with a clear acetate cover placed over each sheet.

For the subjects who classified the digit stimuli, two different types of stimulus lists were used. These were the incongruent digit lists (identical to those employed in Experiment 3) and a single-digit control list. The single-digit control list differed from the one-element control list used in Experiment 3 in that the horizontal location of the digit varied such that its placement on successive displays down the column corresponded to the same alternative horizontal position that would be occupied by the rightmost digit on an incongruent list. Thus, the singledigit control condition provided a very conservative baseline against which possible interference effects brought about by incongruent levels of numerosity could be assessed.

An equivalent set of two types of stimulus displays was given to the subjects who classified number words according to numeric value. These included incongruent word lists identical to those used in Experiment 3, and one-word control lists in which the horizontal location of the word within the column had the same spatial uncertainty as the rightmost word in the incongruent list.

The instructions to subjects were identical to those given to subjects classifying numeric value in Experiment 3, except 
that each trial was initiated by an oral "ready, set, go" signal from the experimenter in place of a visual signal. Subjects pressed a response key upon marking the last stimulus in each list. Feedback about errors but not classification times was given.

On each of the two sessions each subject received two practice blocks of trials, followed by 10 experimental blocks from which data were recorded. Thus, data from a total of 20 blocks of trials were obtained from each subject. The order of the two conditions within a block was simple alternation, with half the subjects beginning with the control list.

\section{Results and Discussion}

For all 10 subjects who classified numeric value of the digit displays and all 10 subjects who classified numeric value of the word displays, mean classification times for the incongruent condition were slower than for the control condition. Thus the 22- and 23-msec interference values shown in Table 7 represent unambiguous evidence for disruption of tapping by incongruent levels of numerosity. Differences between the control and incongruent stimuli were maintained throughout the experiment; the mean classification time across subjects was greater for the incongruent lists on all 20 blocks of trials for both the digit and word stimuli. Thus, under conditions that allow for a substantial amount of practice, there would seem to be a reliable disruptive effect of the display using a response that is structurally more similar to the numerosity dimension.

Manual tapping, therefore, seems to be a response modality for which the pattern of semantic interference is essentially opposite from that found with classifying the same stimuli by oral naming or card sorting. The symbolic component of the display can effectively be ignored, while incongruent levels of numerosity cannot. While the absolute size of the interference effect caused by the numerosity dimension is not overwhelming, it would appear to be maintained even with rather substantial levels of practice.

\section{EXPERIMENT 5: ORAL "TAPPING"}

Despite the fact that the stimulus displays and the logical structure stimulus response mapping were essentially identical throughout the experiments described so far, it might be argued that different information processing demands might arise when

Table 7

Mean Classification (Tapping) Time per Stimulus for Numeric Value in Experiment 4

\begin{tabular}{lcc}
\hline & \multicolumn{2}{c}{ Time } \\
\cline { 2 - 3 } List Type & $\begin{array}{c}\text { Digit } \\
\text { Stimuli }\end{array}$ & $\begin{array}{c}\text { Word } \\
\text { Stimuli }\end{array}$ \\
\hline Control & .566 & .531 \\
Incongruent & .589 & .553 \\
Difference & .023 & .022 \\
\hline
\end{tabular}

scanning a column of stimuli as opposed to sequentially dealing off cards. A somewhat more compelling demonstration of response-context effects on semantic interference would be obtained if fewer details of the task were modified from the procedure followed in Experiment 1. The tasks used in the following experiment, therefore, differ from Experiment 1 (oral naming) in only one detail: Instead of orally saying "one," "two," or "three" to each successive stimulus card, subjects were required to say "duh," "duhduh," or "duhduhduh." Thus the response modality can be viewed as an oral analog of the manual tapping response. As with the manual tapping, there is a close structural resemblance to the numerosity dimension of the stimulus displays, and even though it shares the same physiological output medium as oral naming, the coding processes might be assumed to be more within the semantic domain of numerosity.

\section{Method}

Subjects. Thirty-six undergraduates from the introductory psychology course served in two sessions lasting approximately $1 \mathrm{~h}$ each (on successive days).

Stimulus materials. The same decks of 36 cards as were used in Experiment 1 were used in this experiment.

Procedure. Sixteen subjects were assigned to classify digit displays, eight attending to numerosity and eight attending to numeric value. Twenty subjects were assigned to classify word stimuli, 10 attending to numerosity and 10 to numeric value. The decks given to each of these four groups of subjects were identical to those given to corresponding groups in Experiment 1 except for the following: (1) The oral responses "duh," "duhduh," and "duhduhduh" were substituted for "one," "two," and "three," respectively. (2) Data were collected in two sessions in which subjects received 2 practice and 10 experimental blocks of trials each, thus providing data from a total of 20 classifications of each deck assigned.

\section{Results and Discussion}

Numerosity relevant. In Experiment 1, incongruent variation of numeric value produced a very large interference effect with classifying (by orally naming) the numerosity of the elements in the display. Comparison of Table 8 with Table 1 shows that changing the response to oral tapping reduced the size of the interference effect to where there is only marginal evidence for its existence. The $7-\mathrm{msec} / \mathrm{stimulus}$ difference between classification times for the control and incongruent digit stimuli fell short of statistical significance

Table 8

Mean Classification (Oral Tapping) Time per Stimulus Numerosity Dimension in Experiment 5

\begin{tabular}{lcc}
\hline & \multicolumn{2}{c}{ Time } \\
\cline { 2 - 3 } List Type & $\begin{array}{c}\text { Digit } \\
\text { Stimuli }\end{array}$ & $\begin{array}{c}\text { Word } \\
\text { Stimuli }\end{array}$ \\
\hline Control & .537 & .563 \\
Incongruent & .544 & .573 \\
Difference & .007 & .010 \\
\hline
\end{tabular}


Table 9

Mean Classification (Oral Tapping) Time per Stimulus for Numeric Value Dimension in Experiment 5

\begin{tabular}{lcc}
\hline & \multicolumn{2}{c}{ Time } \\
\cline { 2 - 3 } \multicolumn{1}{c}{ List Type } & $\begin{array}{c}\text { Digit } \\
\text { Stimuli }\end{array}$ & $\begin{array}{c}\text { Word } \\
\text { Stimuli }\end{array}$ \\
\hline One-Item Control & .579 & .593 \\
Three-Item Control & .584 & .585 \\
Incongruent & .611 & .605 \\
Difference & .029 & .016 \\
\hline
\end{tabular}

$[F(1,7)=2.55, \mathrm{MSe}=2.33, \mathrm{p}>.1)$, and only five of the eight subjects classified the incongruent stimuli more slowly. The 10-msec difference observed for the word stimuli was marginally significant $[F(1,7)=7.80$, MSe $=1.74, p<.05]$, yet only 7 out of 10 subjects actually showed the effect. Of major importance, however, is not the existence of interference per se, but that this pattern of data is substantially different from the considerably larger and robust interference effects obtained with oral naming.

Numeric value relevant. The oral tapping response also produced a markedly different pattern of data from either naming or sorting when numeric value was the cue for responding (Table 9). All 8 subjects who classified the digit stimuli and 9 of the 10 subjects who classified the word stimuli produced longer classification times for the incongruent deck than for the mean of the control conditions. The 29- and 16-msec "interference" values are thus statistically reliable, and despite differential room for improvement with practice, there was no Trials by Deck interaction to suggest a washing out of this effect with practice. ${ }^{3}$

To summarize, the pattern of data obtained using the oral tapping response, like the data obtained with manual tapping in Experiments 3 and 4, represents a substantially different pattern than that observed for either oral naming (Experiment 1) or card sorting (Experiment 2). Unlike the data reported by Beller (1975), the present experiments have shown that it is possible to substantially eliminate interference from reading irrelevant semantic information when the response code is sufficiently compatible with the form of the dimension attended. The unwanted reading of symbols, on the other hand, can be shown to produce large interference effects for the very same stimulus sets when a less compatible response code is used. In addition, the present data demonstrated that the physiological aspect of the response modality (i.e., oral vs. manual) is of much less importance in determining compatibility than is relative structural similarity between the stimuli and the response. On the other hand, both the present data and those of Beller (1975) illustrate how modifying the response modality can open the door to new sources of interference; in the present case, the manual and oral tapping responses, which were very efficient for the classification of numerosity, led to interference from irrelevant numerosity when subjects had to classify the symbolic component of the display. Thus, there can be both costs and benefits associated with the changes in attentional states brought about by modifying the motor component of the stimulus response mapping.

\section{GENERAL DISCUSSION}

The present data have provided examples of how the ability to respond to one of two conflicting information sources in a Stroop-like display can be markedly altered by modifying the response modality while other aspects of the task and stimuli remain unchanged. Warren (1972, 1974) has demonstrated a similar change of attentional state, in which the presentation of a priming stimulus causes previously "ignorable" words to disrupt color naming. Both the effects of response preparation, which we have demonstrated, and the effects of semantic priming are consistent with a pathway activation view of selective attention (Posner \& Snyder, 1975). In particular, our data illustrate how common pathways or information processing routines are shared by various components of task performance including the selection of visual information from the array and memory coding operations, as well as motor programs for executing the response.

Interactions between stimulus properties and response set can be demonstrated in a variety of other information processing tasks as well. For example, requiring subjects to respond only to "positive" instances as opposed to both "yes" and "no" responses can substantially reduce the effects of target set size on reaction time in memory scanning tasks (e.g., Egeth, Marcus, \& Bevan, 1972). On the basis of those types of findings, as well as our observed interaction between response modality and Stroop interference, one can make an extremely strong case for the nonindependence of processing stages. Nevertheless, there are a variety of selective attention phenomena that appear to depend almost entirely upon interactions between stimulus properties at an "early" processing stage and are thus less likely to be influenced by modifications of "higher level" task variables (such as the response required of the subject). For example, it is difficult to envision how any manipulation of response factors could improve a subject's ability to "filter" irrelevant variation in the brightness of Munsell color chips while attempting to sort them according to saturation (Garner \& Felfoldy, 1970). "Configural" interactions, such as disrupting the judgment of the right vs. left orientation of a single parenthesis by presenting a contiguous parenthesis that also varies in orientation (Pomerantz \& Garner, 1973), are perhaps another type of selective attention "failure" that reflects perceptual encoding as opposed to semantic resource competition. 
Human performance in any task requiring rapid responses to a complex display is thus potentially constrained by both stimulus-determined perceptual interactions within the display and perceiver-determined interactions at semantic coding or response levels. Separating these two general classes of attentional limitations is not a straightforward process, nor is it clear that they are mutually exclusive sets rather than a continuum (Nickerson, 1978). However, our data have shown how manipulations of response properties while holding the stimuli constant can radically alter one form of perceiver-determined limitation. The systematic manipulation of task variables such as the stimulus response mapping may thus prove to be a useful method for at least partially untangling the various effects of visual distractors in information processing tasks.

\section{REFERENCES}

BELLER, H. K. Naming reading and executing directions. Journal of Experimental Psychology: Human Perception \& Performance, 1975, 1, 154-160.

Broadbent, D. E. Perception and communication. New York: Pergamon Press, 1958.

Deutsch, J. H., \& Deutsch, D. Attention: Some theoretical considerations. Psychological Review, 1963, 70, 80-90.

DYER, F. N. The Stroop phenomenon and its use in the study of perceptual, cognitive and response processes. Memory \& Cognition, 1973, 1, 106-120.

Egeth, H., MaRcus, N., \& Bevan, W. Target-set and responseset interaction: Implications for models of human information processing. Science, 1972, 176, 1447-1448.

Flowers, J. H., \& BlaIR, B. Verbal interference with visual classification: Optimal processing and experimental design. Bulletin of the Psychonomic Society, 1976, 7, 260-262.

Flowers, J. H., \& Dutch, S. The use of visual and name codes in scanning and classifying colors. Memory \& Cognition, 1976, 4, 384-390.

Flowers, J. H., \& Garner, W. R. The effect of stimulus element redundancy on speed of discrimination as a function of state and process limitation. Perception \& Psychophysics, 1971, 9, $158-160$.

Flowers, J. H., \& Stoup, C. M. Selective attention between words, shapes and colors in speeded classification and vocalization tasks. Memory \& Cognition, 1977, 5, 299-307.

GARNER, W. R. The processing of information and structure. Hillsdale, N.J: Erlbaum, 1974.

GARNER, W. R., \& FELFoldy, G. L. Integrality of stimulus dimensions in various types of information processing. Cognitive Psychology, 1970, 1, 225-241.

GREENWALD, A. G. A choice reaction time test of ideomotor theory. Journal of Experimental Psychology, 1970, 86, 20-25. (a)

GREENWALD, A. G. A double stimulation test of ideomotor theory with implications for selective attention. Journal of Experimental Psychology, 1970, 84, 392-398. (b)

Greenwald, A. G., \& Shulman, H. G. On doing two things at once: II. Elimination of the psychological refractory period. Journal of Experimental Psychology, 1973, 101, 70-76.

Hock, H. E., \& Petrasek, J. Verbal interference with perceptual classification: The effect of semantic structure. Perception \& Psychophysics, 1973, 13, 116-120.
KeEle, S. W. Attention demands of memory retrieval. Journal of Experimental Psychology, 1972, 93, 245-248.

LABERge, D. Perceptual learning and attention. In W. K. Estes, Handbook of learning and cognitive processes (Vol. 4). Hillsdale, N.J: Erlbaum, 1976.

MoRTON, J. Categories of interference: Verbal mediation and conflict in card sorting. British Journal of Psychology, 1969, 60, 329-346.

Nickerson, R. S. Comments on W. R. Garner's "Selective attention to attributes and to simuli." Joumal of Experimental Psychology: General, 1978, 107, 452-456.

Norman, D. A. Towards a theory of memory and attention. Psychological Review, 1968, 75, 522-536.

Pomerantz, J. R., \& Garner, W. R. Stimulus configuration in selective attention tasks. Perception \& Psychophysics, 1973, 14, 565-569.

Posner, M. I., \& SNYder, C. R. R. Attention and cognitive control. In R. L. Solso (Ed.), Information processing and cognition: The Loyola symposium. Hillsdale, N.J: Erlbaum, 1975.

Schiffrin, R. M., \& SChNeIDER, W. Controlled and automatic human information Processing II. Perceptual learning, automatic attending, and a general theory. Psychological Review, 1977, 84, 127-190.

STRoop, J. R. Studies of interference in serial verbal reactions. Journal of Experimental Psychology, 1935, 18, 643-662.

Treisman, A. M., \& Fearnley, S. The Stroop test: Selective attention to colours and words. Nature, 1969, 22, 437-439.

Uleman, J. S., \& ReEves, J. A reversal of the Stroop effect through scanning. Perception \& Psychophysics, 1971, 9, 293-295.

WARREN, R. E. Stimulus encoding and memory. Journal of Experimental Psychology, 1972, 94, 90-100.

W ARREN, R. E. Association, directionality, and stimulus encoding. Journal of Experimental Psychology, 1974, 102, 151-158.

WiNDES, J. D. Reaction time for the numerical coding and naming of numerals. Journal of Experimental Psychology, 1968, 78, $318-322$.

\section{NOTES}

1. A small but significant effect of deck type was revealed by analysis of variance $[\mathrm{F}(2,22)=6.75, \mathrm{MSe}=.2 ; \mathrm{F}(2,22)=7.92$, $\mathrm{MSe}=.34 ; \mathrm{p}<.01$ in each case $]$. However, the ordinal position of the means across trials suggest that this effect reflected the number of items per card as opposed to semantic interference. There may have been some degree of performance limitation imposed by visibility restrictions even though the characters were printed in dark ink, and element repetition may have increased opportunities for discriminating a single element in a given unit of time (Flowers \& Garner, 1971). The absolute size of this effect is minimal compared with other effects reported in this study.

2. ANOVA calculations were performed using overall decksorting times, not time per card. Thus values in the tables should be multiplied by 36 for comparison with all the reported error terms. Using the per-card times would have necessitated reporting awkwardly small numbers for MSes.

3. Main effects of deck type were $F(2,14)=11.32$, $\mathrm{MSe}=5.44$, and $\mathrm{F}(2,18)=11.95, \mathrm{MSe}=2.10$, for the digit and word stimuli, respectively; $p<.01$ in each case. Deck by Trials interactions were $\mathrm{F}(38,266)=.94, \mathrm{MSe}=2.03$, and $\mathrm{F}(38,342)=1.41, \mathrm{MSe}=1.05 ; \mathrm{p}>.10$ in each case .

(Received for publication February 22, 1978; revision accepted January $23,1979$. 